\title{
Functional outcome of children treated in intensive care unit
}

\author{
Julije Mestrovic, ${ }^{1}$ Branka Polic, ${ }^{2}$ Marija Mestrovic, ${ }^{3}$ Goran Kardum, ${ }^{4}$ \\ Eugenija Marusic, ${ }^{5}$ Alan Sustic ${ }^{6}$
}

\begin{abstract}
Objective: Outcome of patients is determined not only by severity of illness index, but also by the impact of patients' preadmission comorbid status. Therefore, we aimed at evaluating the outcome of patients treated in a pediatric intensive care unit, with special focus on the group of children with chronic diseases.

Methods: Data were obtained prospectively and outcome was assessed according to the Pediatric Overall Performance Category scale for 449 patients in a pediatric intensive care unit of the Split University Hospital. Functional performance was assessed as the preadmission score and the discharge score in patients with neurodevelopmental disabilities, patients with other chronic diseases, and those without chronic disease.

Results: The discharge functional status was significantly dependent on the preadmission functional status and on predicted mortality. Children with neurodevelopmental disabilities had the significantly worse baseline score and the significantly smaller deterioration of functional morbidity at discharge compared to children with no chronic disease and children with other chronic diseases.

Conclusions: The Pediatric Overall Performance Category scale has proved its applicability in a small intensive care unit, with a heterogeneous population of patients. It should therefore be considered for regular evaluation of health care quality, as a simple and accurate tool. As opposed to other patients, functional status of children with neurodevelopmental disabilities was markedly influenced by their comorbidity. Their preadmission status was worse than the status of other children, and hence could not significantly deteriorate at discharge.
\end{abstract}

J Pediatr (Rio J). 2008;84(3):232-236: Child, critical care, chronic disease, health care quality, outcome assessment.

\section{Introduction}

Health care quality assessment enables identification of differences in health care processes and their benchmarking, with the final aim of improving quality of care. ${ }^{1}$ Outcome of patients basically depends on the severity of illness, which can be classified according to the level of physiologic disturbances of a particular patient caused by the illness index. ${ }^{2}$
However, the impact of the baseline, preexisting chronic illness can sometimes decisively influence the patient's final outcome. $^{3}$

Among the outcome measures, which can be used in pediatric intensive care units (PICU), the Pediatric Overall Performance Category (POPC) scale has been proven to be a simple and valid generic tool. POPC can be used to demonstrate functional morbidity at baseline and at discharge from PICU. ${ }^{4}$ In

1. MD, PhD. Pediatrician. Head, Pediatric Intensive Care Unit, Department of Pediatrics, Split University Hospital, School of Medicine, University of Split, Split, Croatia.

2. Pediatrician. Pediatric Intensive Care Unit, Department of Pediatrics, Split University Hospital, Split, Croatia.

3. MD. Pediatrician, Unit of Pediatric Neurology, Department of Pediatrics, Split University Hospital, Split, Croatia.

4. PhD, BSc. Psychology. Research assistant, Department of Neuroscience, School of Medicine, University of Split, Split, Croatia.

5. MD. Pediatrician, Pediatric Intensive Care Unit, Department of Pediatrics, Split University Hospital, Split, Croatia.

6. MD, PhD. Professor of Anesthesiology, Head, Department of Anesthesiology and ICU, Rijeka University Hospital, Split, Croatia.

No conflicts of interest declared concerning the publication of this article.

Suggested citation: Mestrovic J, Polic B, Mestrovic M, Kardum G, Marusic E, Sustic A. Functional outcome of children treated in intensive care unit. J Pediatr (Rio J). 2008;84(3):232-236.

Manuscript received Dec 10 2007, accepted for publication Feb 202008.

doi:10.2223/JPED.1779 
this study we tried to define the influence of the preadmission functional status of patients treated in PICU on their condition at discharge.

\section{Methods}

PICU of the Split University Hospital is a seven-bed, multidisciplinary unit, with an additional three step-down beds where children who need chronic ventilation are monitored. The unit admits all children from newborns up to and including 18 years of age. All medical and surgical (but excluding children with congenital heart diseases) patients and newborns transported by "two-way transport" from other towns are admitted to the unit.

From June 2002 through July 2004, data was obtained prospectively from 493 survivors aged $\leq 18$ years, but excluding preterm infants. Demographic data, such as age, sex, and the need for ventilatory support were collected. Each patient's main reason for PICU admission was recorded in accordance with the Australia and New Zealand Pediatric Intensive Care Registry (ANZPIC Registry) of diagnostic codes. ${ }^{5}$ Parameters for the determination of the pediatric index of mortality (PIM) score were recorded during the first hour of admission, and mortality risk for each patient was calculated according to the equations developed and published by the PIM Study Group. ${ }^{6,7}$ Outcome of patients was assessed according to the POPC scale for 449 patients. POPC is a six-point scale ranging from 1 (normal) to 6 (dead), with interim point representing progressively greater functional impairment. Each scale category is accompanied by age-appropriate operational definitions. ${ }^{4}$ Functional performance of patients was assessed as the baseline, preadmission score (bPOPC), prior to index disease, based on the interview with each child's parent. The discharge functional score (dPOPC) was evaluated before transfer of patients from PICU by the same physician (J.M. or B.P.) who recorded the preadmission score. Functional condition deterioration was expressed as the change in POPC score from admission to discharge ( $\triangle P O P C)$. Chronic health conditions were defined according to the Maternal and Child Health Bureau (MCHB) as ongoing physical, developmental, behavioral, or emotional conditions that required health and related services of a type and amount in excess of what is usually beyond that generally required by children. ${ }^{8}$ Neurodevelopmental disabilities were defined according to the adaptation of Crocker's categorization of developmental disabilities and mental retardation. ${ }^{9}$ The study protocol was approved by the Hospital's Ethics Committee. Demographic data, such as age, sex, need for ventilatory support and length of stay, were collected. The chi-square test and analysis of variance (ANOVA) using Tukey's post hoc test, along with Kruskal-Wallis and Mann-Whitney tests were used in statistical analysis, with results considered significant at $95 \%(p<0.05)$.

\section{Results}

Median age of our patients was 36.5 months. There were $55 \%$ male patients, $28 \%$ of patients were ventilated, and $34 \%$ were surgical. The admissions were elective for $17 \%$ patients, and $24 \%$ of admissions were recovery.

Respiratory diagnoses (25.3\%) and injuries (20\%) accounted for the majority of main reasons for admission, followed by neurological (14.2\%), postoperative (12\%) and cardiac $(4.8 \%)$ diagnoses, while $23.7 \%$ of diagnoses were miscellaneous.

The number of patients with good overall performance decreased from $63 \%$ at admission to $42.3 \%$ at discharge (Figure 1). The largest increase at discharge was exhibited by the group with mild overall disability $(9.8-22.6 \%)$. dPOPC was significantly dependent on bPOPC $(p<0.001)$.

The correlation between PIM and dPOPC score demonstrated the significant influence of severity of acute illness at admission on functional status of patients at discharge ( $p<$ 0.001) (Figure 2).

In our population there were 75 children with neurodevelopmental disabilities and 96 children with other chronic diseases. Children with neurodevelopmental disabilities had significantly worse baseline scores $(p<0.001)$ (Table 1$)$. In $113(25 \%)$ patients the dPOPC was worse than the bPOPC. Functional morbidity deterioration was significantly less frequent in children with neurodevelopmental disabilities $(p<$ 0.001 ), compared to the other two groups of patients (Table 2).

\section{Discussion}

The associations of discharge function with mortality risk, length of stay, and diagnostic groups of patients treated in PICU have already been demonstrated. ${ }^{4,10}$

The important determinant of outcome is basic, preadmission functional level. ${ }^{3}$ Despite its obvious importance, the impact of premorbid disease has been rarely evaluated. However, assessment of functional level at discharge, as well as health-related quality of life of children after treatment in intensive care units, points to the significant correlation between the preadmission function of patients and their short-term or long-term outcome. ${ }^{3,4,10,11}$ Our results showed the significant dependence of the discharge functional status on the baseline function of patients. This observation inspired us to undertake a more detailed analysis of populations of patients included in the study. Since previous results indicated that children with neurodevelopmental disabilities represent the particularly susceptible group, this group of patients was further analyzed separately. ${ }^{12}$ The data collected from parents at admission indicate the significantly impaired baseline functional status of our patients with neurodevelopmental disabilities compared to children with no chronic disease and children with other chronic diseases. It has been broadly documented that children with neurological disabilities are burdened with severe physical and cognitive difficulties, which can significantly impair their everyday life. ${ }^{13-17}$ 


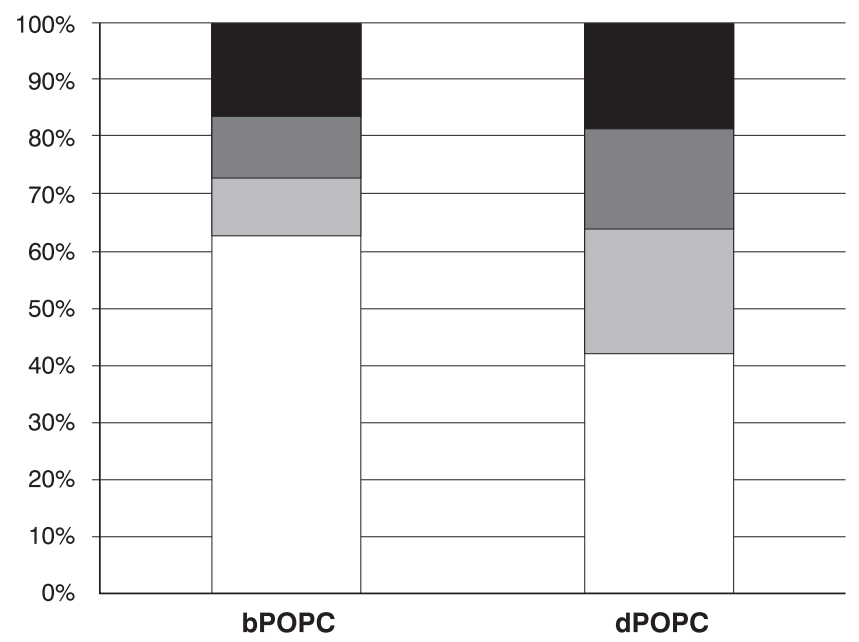

Severe disability $\square$ Moderate disability $\square$ Mild disability $\square$ Good

$\mathrm{bPOPC}=$ baseline functional status; $\mathrm{dPOPC}=$ functional outcome at discharge.

Figure 1 - Classification of baseline functional status and functional outcome at discharge of children treated in the pediatric intensive care unit of Split University Hospital

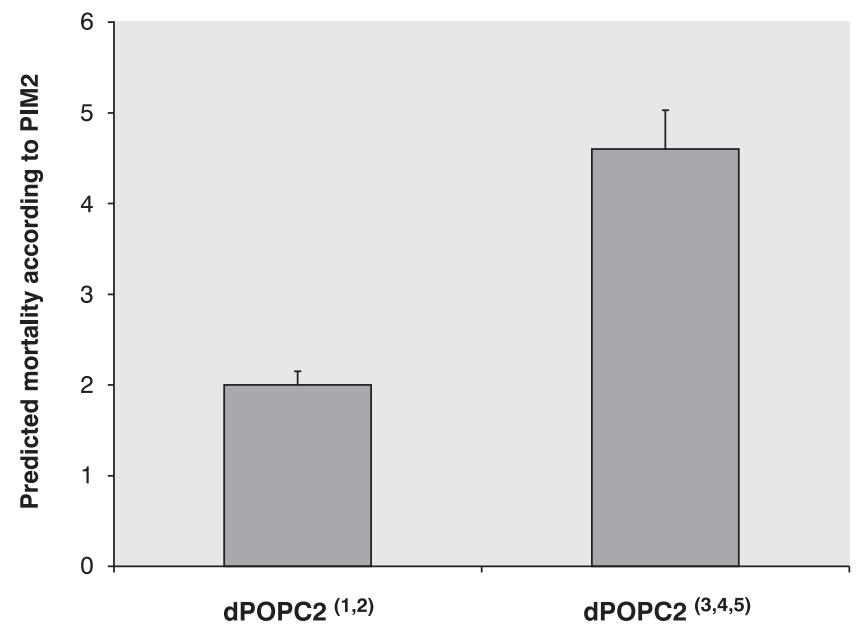

$\mathrm{dPOPC}=$ functional outcome at discharge.

Figure 2 - Predicted mortality calculated according to pediatric index of mortality in patients with good performance and mild overall disability ${ }^{1,2}$ and in patients with moderate or severe disability and with coma/vegetative state ${ }^{3-5}$ at discharge

Children with neurodevelopmental disabilities are significantly more susceptible to serious acute diseases due to their chronic condition. ${ }^{13,18}$ Therefore, strong influence of patients' comorbidity further aggravates clinical condition caused by acute disease. As a consequence, patients with neurodevelopmental disabilities need to be treated in intensive care units more often than other patients. ${ }^{13}$ In spite of the need for more serious treatment, the functional status of children with neurodevelopmental disabilities at their discharge from PICU was not significantly deteriorated. It remained similar to their baseline functional status. The low baseline function is the impact of comorbidity, which explains the observed mild changes of outcome at discharge, and rare deteriorations of the functional level in our patients.

This study certainly suffers from a clear limitation, namely, our unit is small. The differences in patients' outcomes in small units can be expected to be unconvincing. The small and 
Table 1 - Proportions of categories of functional performance before admission in patients with no chronic disease, neurodevelopmental disability, and other chronic disease

\begin{tabular}{lcccc}
\hline Group & bPOPC* & bPOPC $^{+}$ & $\boldsymbol{\Lambda}^{\mathbf{2}}$ & $\mathbf{p}$ \\
\hline No chronic disease & 276 & 5 & 308.21 & $<0.001$ \\
$\begin{array}{l}\text { Neurodevelopmental } \\
\text { disability }\end{array}$ & 26 & 49 & 308.21 & $<0.001$ \\
Other chronic disease & 77 & 19 & 308.21 & $<0.001$ \\
Total & 376 & 73 & 308.21 & $<0.001$ \\
\hline
\end{tabular}

bPOPC = baseline functional score.

* Good performance, mild and moderate overall disability.

+ Severe disability, and with coma/vegetative state.

Table 2 - Functional morbidity deterioration in patients with no chronic disease, neurodevelopmental disability, and other chronic disease

\begin{tabular}{lcccc}
\hline Group & No & $\mathbf{A P}$ & $\boldsymbol{\Lambda}^{\mathbf{2}}$ & $\mathbf{p}$ \\
\hline No chronic disease & 278 & 99 & 30.86 & $<0.001$ \\
$\begin{array}{l}\text { Neurodevelopmental } \\
\text { disability }\end{array}$ & 75 & 3 & 30.86 & $<0.001$ \\
Other chronic disease & & & 30.86 & $<0.001$ \\
Total & 96 & 11 & 30.86 & $<0.001$
\end{tabular}

$\Lambda P=$ deterioration of functional morbidity .

biased sample of patients could make the comparison between the results obtained in other units and the results of the unit in the study deprived of reliability. However, the comparability of results of our study is supported by the structure heterogeneity of included patients. The heterogeneous case-mix makes the pattern of patients more adequate for analysis in relation to the studies with much larger populations. ${ }^{4,10}$

Hence, in spite of differences between the units in populations of patients managed and available specialties, the POPC scale has shown to be a simple and accurate tool for assessment of outcome in intensive care units. It is, therefore, advisable to use it regularly as a means of evaluating health care quality in pediatric intensive care.

\section{References}

1. Fiser DH. Outcome evaluations as measures of quality in pediatric intensive care. Pediatr Clin North Am. 1994;41:1423-38.

2. Marcin JP, Pollack MM. Review of the methodologies and applications of scoring systems in neonatal and pediatric intensive care. Pediatr Crit Care Med. 2000;1:20-7.

3. Greenfield S, Aronow HU, Elashoff RM, Watanabe D. Flaws in mortality data. The hazards of ignoring comorbid disease. JAMA. 1988;260:2253-5.
4. Fiser $\mathrm{DH}$. Assessing the outcome of pediatric intensive care. J Pediatr. 1992; 121:68-74.

5. Slater A, Shann F, McEniery J. The ANZPIC registry diagnostic codes: a system for coding reasons for admitting children to intensive care. Intensive Care Med. 2003;29:271-7.

6. Slater A, Shann F, Pearson G; Paediatric Index of Mortality (PIM) Study Group. PIM2: a revised version of the Paediatric Indeks of Mortality. Intensive Care Med. 2003;29:278-85.

7. Mestrovic J, Kardum G, Polic B, Omazic A, Stricević L, Sustic A. Applicability of the Australian and New Zealand Paediatric Intensive Care Registry diagnostic codes and Paediatric Index of Mortality 2 scoring system in a Croatian paediatric intensive care unit. Eur J Pediatr. 2005;164:783-4.

8. McPherson $M$, Arango $P$, Fox $H$, Lauver C, McManus M, Newacheck PW, et al. A new definition of children with special health care needs. Pediatrics. 1989;102:137-40.

9. Crocker AC. The causes of mental retardation. Pediatr Ann. 1989; 18:623-9.

10. Fiser DH, Tilford JM, Roberson PK. Relationship of illness severity and length of stay to functional outcomes in the pediatric intensive care unit: a multi-institutional study. Crit Care Med. 2000;28:1173-9.

11. Morrison AL, Gillis J, O'Connell AJ, Schell DN, Dossetor DR, Mellis C. Quality of life of survivors of pediatric intensive care. Pediatr Crit Care Med. 2002;3:1-5.

12. Taylor A, Butt W, Ciardulli M. The functional outcome and quality of life of children after admission to an intensive care unit. Intensive Care Med. 2003;29:795-800. 
13. Mestrovic J, Kardum G, Polic B, Mestrovic M, Sustic A, Markic J, et al. The influence of chronic health conditions on susceptibility to severe acute illness of children treated in PICU. Eur J Pediatr. 2006;165:526-9.

14. Pirpiris M, Gates PE, McCarthy JJ, D'Astous J, Tylkowksi C, Sanders JO, et al. Function and well-being in ambulatory children with cerebral palsy. J Pediatr Orthop. 2006;26:119-24.

15. Seddon PC, Khan Y. Respiratory problems in children with neurological impairment. Arch Dis Child. 2003;88:75-8.

16. Hutton JL, Pharoah PO. Effects of cognitive, motor, and sensory disabilities on survival in cerebral palsy. Arch Dis Child. 2002;86: 84-9.

17. Ireyes HT. Epidemiology of childhood chronic illness: issues in definitions, service use, and costs. In: Koot HM, Wallander JL, editors. Quality of life in child and adolescent illness. New York: Taylor \& Francis; 2001. p.123-50.
18. Dosa NP, Boeing NM, Kanter RK. Excess risk of severe acute illness in children with chronic health conditions. Pediatrics. 2001; 107:499-504.

Correspondence:

Julije Meštrović

Pediatric Intensive Care Unit

Department of Pediatrics, Split University Hospital

Spinčićeva 1

21000 - Split - Croatia

Tel.: + 385 (0) 21.556686

Fax: +385 (0) 21.556590

E-mail: julije.mestrovic@st.t-com.hr 